\title{
Avaliação de dois métodos de diagnóstico por imagem como preditores do tempo de ventilação mecânica em pacientes submetidos a transplante hepático
}

\section{Diogo Silva Lira*, Ilka de Fátima Santana Ferreira Boin, Paulo Leão Perin, Luiz Cláudio Martins.}

\section{Resumo}

As alterações pulmonares, devido ao estado inflamatório sistêmico, possuem grande impacto no prognóstico e sobrevida dos pacientes com doença hepática avançada. Esta pesquisa teve como escopo correlacionar as alterações pulmonares visualizadas na ultrassonografia (US) com as alterações visualizadas na tomografia computadorizada de alta resolução (TC) - considerada padrão-ouro para avaliação pulmonar - , a gravidade da doença hepática, alterações da espirometria, tempo de ventilação mecânica e tempo de internação na Unidade de Terapia Intensiva (UTI). Dentre os pacientes estudados, não houve diferença significativa entre US e TC para avaliação de óbito e desfechos póstransplante, o que fortalece o ganho de importância da avaliação pré-operatória com a US, um exame de menor custo, menos invasivo e de maior facilidade de realização, além de ser mais sensível para detectar congestão pulmonar, um possível fator que reduz as chances de realização de transplante hepático, por aumentar o risco cirúrgico.

\section{Palavras-chave:}

Ultrassonografia, tomografia computadorizada, transplante hepático.

\section{Introdução}

As alterações pulmonares possuem grande impacto na evolução e sobrevida dos pacientes com acometimento hepático, sendo assim, importante sua avaliação durante 0 período preparatório para 0 transplante hepático.

O exame considerado padrão-ouro para avaliação morfológica pulmonar é a tomografia computadorizada de alta resolução (TC) do tórax, possuindo uma sensibilidade de $95 \%$ e uma especificidade próxima aos $100 \%$, embora sendo invasivo e de alto custo.

A ultrassonografia pulmonar (US), devido ao seu menor custo e maior facilidade de realização, tem se despontado como importante método na avaliação pulmonar, exercendo impacto nas condutas e nos diagnósticos em pacientes internados na Unidade de Terapia Intensiva sob suspeita de doença pulmonar aguda ${ }^{1}$.

Esse estudo procurou comparar os resultados dessas duas modalidades de exames de imagem, realizados no período pré-operatório dos pacientes na fila do transplante hepático, e avaliar a relação com o prognóstico e a sobrevida no pós-operatório.

\section{Resultados e Discussão}

Foi realizado um estudo transversal a partir da busca de dados de pacientes com diagnóstico de hepatopatia e indicação de transplante hepático em seguimento no ambulatório de transplante hepático do Hospital de Clínicas da Universidade Estadual de Campinas.

Foram selecionados 27 pacientes (22 do sexo masculino e 5 do feminino), que preencheram o critério de ser submetido ao transplante.

Posteriormente, a fim de correlacionar os achados de imagem (exames realizados previamente ao transplante) com o prognóstico e sobrevida póstransplante, foi realizada a análise de cada tipo de alteração tomográfica e ultrassonográfica com os seguintes desfechos: tempo de ventilação mecânica, tempo de internação na Unidade de Terapia Intensiva (UTI), tempo de internação hospitalar e óbito.

Tabela 1. Correlação TC x Desfecho

\begin{tabular}{|c|c|c|}
\hline & $\begin{array}{c}\text { TC normal } \\
(\mathrm{N}=14)\end{array}$ & $\begin{array}{c}\text { TC alterada } \\
(\mathrm{N}=13)\end{array}$ \\
\hline TVM (dias) & $17 \pm 39,74$ & $1,46 \pm 0,96$ \\
\hline TUTI (dias) & $14,78 \pm 29,57$ & $4,07 \pm 2,01$ \\
\hline TIH (dias) & $27,28 \pm 42,99$ & $7,92 \pm 5,52$ \\
\hline Óbito & $18,75 \%$ & $23 \%$ \\
\hline
\end{tabular}

Tabela 2. Correlação US x Desfecho

\begin{tabular}{|c|c|c|}
\hline & $\begin{array}{c}\text { US normal } \\
(\mathrm{N}=10)\end{array}$ & $\begin{array}{c}\text { US alterada } \\
(\mathrm{N}=17)\end{array}$ \\
\hline TVM (dias) & $14,2 \pm 40,34$ & $7,29 \pm 21,45$ \\
\hline TUTI (dias) & $4,9 \pm 4,33$ & $12,41 \pm 27,0$ \\
\hline TIH (dias) & $19,6 \pm 38,9$ & $17,0 \pm 28,78$ \\
\hline Óbito & $10 \%$ & $29,40 \%$ \\
\hline
\end{tabular}

TVM: Tempo de Ventilação Mecânica

TUTI: Tempo de internação na Unidade Terapia Intensiva

TIH: Tempo de Internação Hospitalar

TC: Tomografia Computadorizada de Alta Resolução do tórax US: Ultrassonografia pulmonar

$\mathrm{N}$ : número de pacientes

TC alterada vs US alterada para os desfechos estudados: valor de $\mathrm{p}<$ 0,05 .

\section{Conclusões}

A partir dos resultados, observou-se que não houve diferença significativa dentre os exames para o desfecho de tempo de ventilação mecânica, tempo de internação em UTI e tempo de internação hospitalar. Dessa forma, por ser um exame de maior facilidade de realização, com menor custo, menos invasivo e, portanto, mais benéfico ao paciente, justifica-se o ganho de importância da US no uso da avaliação pré-operatória, em substituição à TC. Identificou-se também maior tempo de ventilação e de mortalidade nos pacientes com maior escore CHILD e com ascite, corroborando sua importância prognóstica.

\section{Agradecimentos}

Ao PIBIC e ao Dr. Helymar C. Machado (estatístico).

\footnotetext{
${ }^{1}$ Xirouchaki N1, Kondili E, Prinianakis G, Malliotakis P, Georgopoulos D. Impact of lung ultrasound on clinical decision making in critically ill patients. Intensive Care Med. 2014.
} 